\title{
Kardinal Die Stimme erheben \\ Karl Lehmann Würdigung von Medienbischof Dr. Hermann \\ Josef Spital durch den Vorsitzenden der \\ Deutschen Bischofskonferenz
}

Runde Geburtstage sind Lebensabschnitte. Ein 75. Geburtstag markiert dabei für Priester einen besonderen Moment, da mit diesem Datum in der Regel der wohlverdiente Ruhestand beginnt. Bischof Dr. Hermann Josef Spital hat am 31. Dezember 2000 diese Wegmarke erreicht. Es ist nicht nur ein Einschnitt in seiner persönlichen Biographie, sondern ganz besonders auch für die Deutsche Bischofskonferenz. Wir schulden Hermann Josef Spital großen Dank.

Nach seiner Bischofsweihe 1980 war er zunächst Vorsitzender der Liturgiekommission unserer Konferenz, bevor er 1989 den Vorsitz des verstorbenen Rottenburg-Stuttgarter Bischofs Dr. Georg Moser in der Publizistischen Kommission der Deutschen Bischofskonferenz übernahm. Über ein Jahrzehnt hat Hermann Josef Spital in dieser Verantwortung gewirkt. Höhen und Tiefen der Verlagslandschaft, die rasante Entwicklung auf dem Markt der neuen Medien, insbesondere Hörfunk und Fernsehen, hat er miterlebt. Für dieses Engagement sei ihm an dieser Stelle ganz besonders gedankt.

Es wäre müßig, die einzelnen Bereiche, in denen sich Hermann Josef Spital auf dem Mediensektor bewegt hat, hier aufzuzählen. Dennoch scheint es mir angebracht, auf einige Fakten hinzuweisen. So ist unter seiner Federführung die Gemeinsame Erklärung der Deutschen Bischofskonferenz und des Rates der Evangelischen Kirche, "Chancen und Risiken der Mediengesellschaft" (1997) entstanden. Den Kommissions. vorsitz übernahm Spital in einer Zeit, als der "Weltbild Verlag" wirtschaftlich schwierige Zeiten durchlebte. Unter ihm gelang die Konsolidierung und nachher sehr positive Entwicklung des Unternehmens.

Weitere wichtige Dokumente, die in der Zeit seiner Verantwortung entstanden und die das kirchliche Medienengagement in den kommenden Jahren bestimmen werden, waren beispielsweise "Multimedia. Der Wandel der Informationsgesellschaft“ (1995) und „Positionierung der katholischen Kirche in den elektronischen Medien" (2000).

Neben dieser Verantwortung für den nationalen Bereich stand sein Wirken auf internationaler Ebene. Seit gut zehn Jahren gehört Spital als Mitglied dem Päpstlichen Rat für die sozialen Kommunikationsmittel an. Hier brachte er die Überlegungen der deutschen Kirche in so richtungweisende Dokumente ein wie die "Pastoralinstruktion ,Aetatis novae“" 
(1992) und in Grundsatzbestimmungen wie "Ethik in der Werbung" (1997) und „Ethik in der sozialen Kommunikation“ (2000).

Hermann Josef Spital hat sich in seiner Amtszeit stets um Ausgleich bemüht. Dabei war er ein Verfechter für den Erhalt der Kirchenzeitungen - in welcher redaktionellen und wirtschaftlichen Form auch immer -, weil sie für ihn Informationsorgane sind, die nicht wie ein Großteil der medialen Wirklichkeit den Schwerpunkt auf das Schrille und Sensationelle legen. Die Vielfalt kirchlicher Presse war für Spital Ausdruck der Lebendigkeit unserer Kirche. Diese Lebendigkeit - wiederholte er häufig - gelte es zu erhalten.

Ohne Frage lag Hermann Josef Spitals mediale Passion in einem eher traditionsreichen Medium, dem Buch. Wo er konnte, hat er versucht, sich für das Buch stark zu machen. Er war ein Verfechter des gesicherten Mittelstandes im katholischen Verlagswesen. Sorge bereitete ihm das Schwächerwerden des konfessionellen Buchhandels. In seine Amtszeit fällt die Fusion von zwei katholischen Medienverbänden zum neuen, seit dem Jahr 2000 existierenden „Katholischen Medienverband“ - Bischof Spital wusste, wann die Zeichen der Zeit zu einschneidenden aber notwendigen Maßnahmen gegeben waren.

Das gedruckte Wort hat Spital Zeit seines Lebens geprägt. In der Bischofskonferenz, im Bistum Trier aber auch weit dariber hinaus sind seine Publikationen, insbesondere die Hirtenbriefe der vergangenen Jahre auf ein lebhaftes Echo gestoßen. Mit dem Wort des Völkerapostels, "Prüft alles und behaltet das Gute" (1 Thess 5,21) war es Spital, der Literatur zu differenzieren wusste. Wo es ihm gegeben war, versuchte er für das Lesen und das Schriftwort zu werben. 1999 stellte er in seiner Erklärung "Leselust und Lesenutzen" fest: „Auf dem Markt der Medien wird das Buch seinen Platz nur dann behaupten, wenn wir seinen Wert begreiflich machen können ... An diesem Kommunikationsprozess müssen wir uns alle beteiligen, denn es geht um eine Frage der Kultur und des Wissens."

Für Spital ist das Lesen eine lebensnotwendige Herausforderung: Der Mensch ist auf der Suche nach sich selbst. Um dorthin zu finden, ist es notwendig, Erfahrungen zu machen. Solche Erfahrungen kann es im Leben und in der Literatur geben. Erfahrungen spiegeln dann also menschliches Handeln wider. Man kann nicht leben in einer Erfahrung, die nicht Geschichte wird - lautet die Philosophie Spitals für das gedruckte Wort. Moderne Literatur ist für ihn ein Lebensbestandteil, um so die Fragen der Menschen von heute wahrzunehmen. Wer ihn kennt, weiß, dass er ein Anhänger eines jeden neuen Romans von Matthias Mander ist, weil er für ihn Wirklichkeit zur Sprache bringt. Um Erfahrungen zu ermöglichen und Räume dafür zu schaffen, war es Bischof Spital daher ein Anliegen, 
das vom Borromäusverein 1999 konzipierte „Projekt Literarische Kompetenz" ins Rollen zu bringen.

Im März 2000 haben wir in der Frühjahrsvollversammlung einen Studienhalbtag zu „Grundsätzlichen Fragen des kirchlichen Engagements in den Medien" durchgeführt. Dabei betonte Bischof Spital seine innerste Überzeugung, dass gerade die Kirche in der sich rasant entwickelnden Informations- und Mediengesellschaft Zeugin, Partnerin und Betroffene des medialen Wandels sei. Als Vorsitzender der Publizistischen Kommission hat er der Kirche einen wichtigen Dienst geleistet: sich nicht in ein Ghetto zurückzuziehen, sondern mit einem guten Maß an Selbstbewusstsein auf diesem Feld nach vorne zu schauen. Dabei war Hermann Josef Spital nicht ein Medienprophet, dem Visionen oder das nötige Bewusstsein für eine realistische Einschätzung fehlten. Keineswegs, denn gerade er wusste um die schwierigen finanziellen und personellen Ressourcen im kirchlichen Medienengagement. Sein Plädoyer hieß daher "gerechter Lastenausgleich" und Aufmerksamkeit für das, was nicht nur Mode, sondern die Herausforderung der Stunde war. Dabei hat Hermann Josef Spital seine Umgebung durch die ihm eigene Weltoffenheit beeindruckt. Seine leidenschaftliche Zuneigung zu allen Methoden und Formen der Kommunikation waren der Maßstab seines Handelns im medialen Bereich. Er war im Medienumgang der Mann deutlicher, aber nicht lauter Worte. Er ist fest überzeugt, dass es notwendig sei, die Stimme in der Öffentlichkeit $z u$ erheben. Rundfunkräte seien dazu da, auch dann die Meinung zu sagen, wenn man nicht mehrheitsfähig sei. „Die Stimme muss man erheben, auch wenn es nicht immer ein Erfolg ist", stellte Spital einmal in einem Interview fest. Die Grundlage des medialen Engagements hieß dabei für ihn immer, auf dem Fundament der Frohen Botschaft Jesu zu stehen. Sie hat ihn bis heute als Christ und in seinem priesterlichen Wirken erfillt, sie war zugleich immer Maßstab beim Fragen, wie Verkündigung in den kirchlichen und säkularen Medien angesichts knapp bemessener Freiräume in Zeitungen und enger Sendezeiten im Rundfunk realisiert werden könne.

Jetzt darf sich Bischof Hermann Josef Spital ein wenig zurücklehnen, sich weiter dem Medium Buch und vor allem den von ihm geschätzen neutestamentlichen hermeneutischen Forschungen widmen. Im Namen der Deutschen Bischofskonferenz, aber auch ganz persönlich danke ich dem Vorsitzenden der Publizistischen Kommission, dem Bischof und Priester Hermann Josef Spital, dem Mitbruder für sein unermüdliches Wirken, seine Feinfühligkeit im Umgang mit dem Wort in den Medien und seine Bereitschaft, Neues im Medienfeld auszuprobieren, um den Dienst einer kommunikativen Kirche nach innen und außen zu erfüllen. Für sein segensreiches Wirken in der Medienkommission gilt ihm ein 
besonderer Dank, den ich mit der Hoffnung verbinde, dass das dort $\mathrm{Be}$ gonnene fortgeführt wird. Bischof Hermann Josef Spital hat der Öffentlichkeit gezeigt, dass er mehr als manch anderen mit Blick auf die Informationsgesellschaft das Pauluswort "Priff alles und behaltet das Gute" zu nutzen wusste. Ad multos annos!

Kardinal Karl Lehmann

Vorsitzender der Deutschen Bischofskonferenz 